CHAPTER 20

\title{
RESEARCH TO IMPROVE ARTEMISININ PRODUCTION FOR USE IN THE PREPARATION OF ANTI-MALARIAL DRUGS
}

\author{
HARRO BOUWMEESTER ${ }^{\#}$, CINZIA BERTEA, \\ JAN-WILLEM DE KRAKER, FRANCEL VERSTAPPEN AND \\ MAURICE FRANSSEN

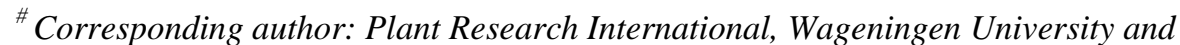 \\ Research Centre, P.O. Box 16, 6700 AA Wageningen, The Netherlands. \\ E-mail: harro.bouwmeester@wur.nl
}

\begin{abstract}
An important group of antimalarial drugs consists of the endoperoxide sesquiterpene lactone artemisinin and its semi-synthetically prepared derivatives. Because of the development of resistance against other malarial drugs, the demand for artemisinin has rapidly increased during the past decade. However, the supply of artemisinin is troublesome as neither total nor semi-synthesis are economically feasible and the only plant species known to produce artemisinin, Artemisia annua L., contains only low amounts of this compound. For a directed strategy to improve artemisinin production, one of the first requirements is to have insight into the regulation of its biosynthesis. Surprisingly, until some years ago hardly anything was known about the biosynthesis of artemisinin, particularly about the early enzymatic steps leading to (dihydro)artemisinic acid. To elucidate this important missing part in the pathway, we have analysed the terpenoids in A. апnиa leaves and the presence of sesquiterpene synthases, the enzyme class that should theoretically catalyse the first step in the formation of artemisinin. This led to the discovery of amorpha-4,11-diene synthase, which indeed catalyses the first step in artemisinin biosynthesis and for which the corresponding gene was cloned shortly afterwards. A subsequent search for oxidized derivatives of amorpha-4,11-diene - which could be postulated to be intermediates en route to artemisinin - revealed the presence of artemisinic alcohol, dihydroartemisinic alcohol, artemisinic aldehyde, dihydroartemisinic aldehyde and dihydroartemisinic acid. We also demonstrated the presence of the biosynthetic enzymes amorpha-4,11-diene hydroxylase, artemisinic alcohol dehydrogenase, artemisinic aldehyde reductase and dihydroartemisinic aldehyde dehydrogenase in A. annua. From these results, we conclude that the early steps in artemisinin biosynthesis involve: cyclization of farnesyl diphosphate by amorpha-4,11-diene synthase to amorpha-4,11-diene, hydroxylation of amorpha-4,11diene to artemisinic alcohol, oxidation of artemisinic alcohol to artemisinic aldehyde, reduction of the C11-C13 double bond yielding dihydroartemisinic aldehyde, and oxidation of dihydroartemisinic aldehyde to dihydroartemisinic acid. During this biochemical research we have also managed to develop a trichome isolation protocol which has not only accelerated our biochemical work but also enables us to clone - in a very directed way - the genes that encode the enzymes responsible for artemisinin production. This should in the next few years result in several approaches to boost artemisinin production.
\end{abstract}

275

R.J. Bogers, L.E. Craker and D. Langer (eds.), Medicinal and Aromatic Plants, 275-290. (C) 2006 Springer. Printed in the Netherlands 
Keywords: Artemisia annua; Asteraceae; artemisinin; biosynthetic pathway; sesquiterpenoids; biosynthesis; plant secondary metabolites

\section{INTRODUCTION}

Malaria is an infectious disease caused by protozoa of the genus Plasmodium, which are carried by mosquitoes of the genus Anopheles. The most severe form of malaria, malaria tropica, is responsible for 300-500 million clinical cases each year, of which about $90 \%$ occur in Africa. It is estimated that malaria causes between 1.5 and 3 million deaths per year, mainly African children (Butler et al. 1997; Rinaldi 2004). In the past, the fight against malaria was based on two strategies: (i) The extermination of the mosquito vector with pesticides such as DDT. This approach was stopped because of the emergence of mosquito resistance against DDT and the undesirable side effects on the reproduction of animals and its persistence in food chains. As a consequence the occurrence of malaria is now spreading. (ii) The largescale use of quinine and chloroquine for the treatment of patients with malaria and as prophylaxis. This has evoked resistance of the protozoa against these drugs, making the fight against malaria more difficult; malaria now forms an increasing problem in the (sub)tropics. Moreover, it is anticipated that due to global warming, malaria will spread to regions such as Southern Europe and the USA. Therefore, there is a continuous search for new remedies against malaria (Rathore et al. 2005; Wright 2005).

In traditional Chinese medicine, the herb Artemisia annua L. (sweet wormwood; Asteraceae) is used since a long time. The earliest mention of this herb dates back to $168 \mathrm{BC}$ in the ancient recipes found in the tomb of the Mawangdui Han dynasty (Klayman 1985). Its antimalarial activity was described as early as 1596 by $\mathrm{Li}$ Shizhen in his Ben Cao Gang Mu (Compendium of Materia Medica) (Klayman 1985). The active principle was isolated and identified as artemisinin in 1972. Artemisinin (also known as qinghaosu) appeared to be a sesquiterpene lactone endoperoxide (see Figure 1). Since then there has been a tremendous scientific and commercial interest in this rediscovered antimalarial compound. Based on this secondary plant metabolite, several synthetic derivatives such as artemether, arteether, artesunic acid and artelinic acid have been produced, which are effective against multidrug-resistant Plasmodium falciparum strains, the organism responsible for malaria (Ridley 2002; Wallaart et al. 1999b).

A. annua is a cosmopolitan species, growing wild in many countries, e.g. in China and Vietnam, the Balkan, the former Soviet Union, Argentina and Southern Europe (Van Geldre et al. 1997), and large differences exist in artemisinin content between different varieties of A. annua (Delabays et al. 1993; Woerdenbag et al. 1993). A substantial increase in the content of artemisinin would be required to make artemisinin available on a large scale also to the people in the Third World. Selection for high-producing lines and traditional breeding, and research on the effects of environmental conditions and cultural practices could perhaps lead to an improvement of artemisinin content (Delabays et al. 1993; Ferreira et al. 1995; Gupta et al. 1996). A biotechnological approach could be another way to increase artemisinin content in A. апnиа. Because chemical synthesis of artemisinin is an 

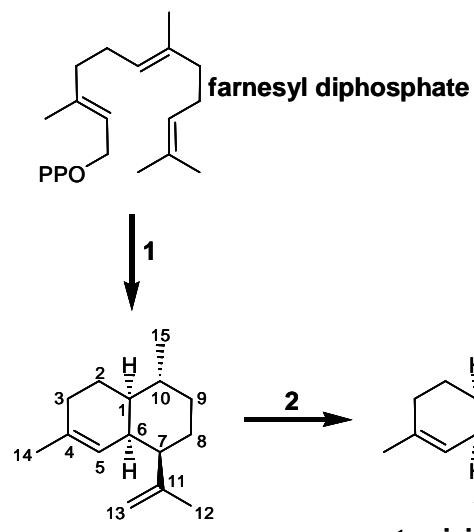

amorpha-4,11-diene
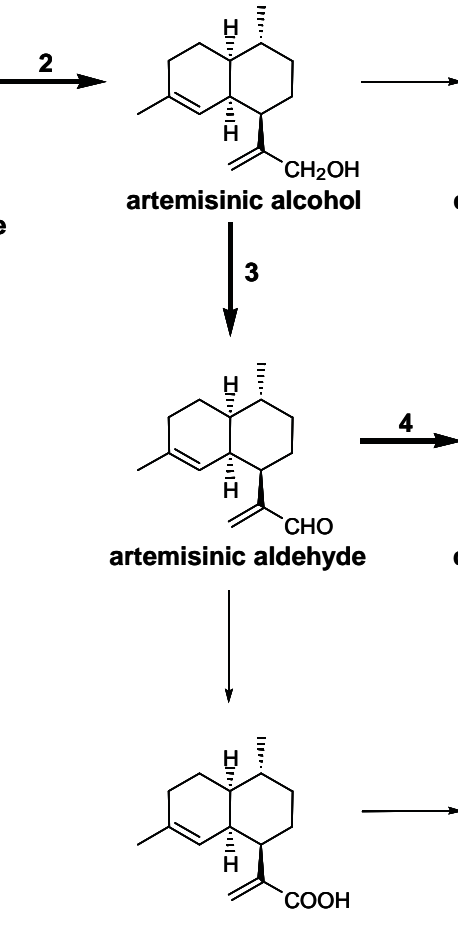

artemisinic acid
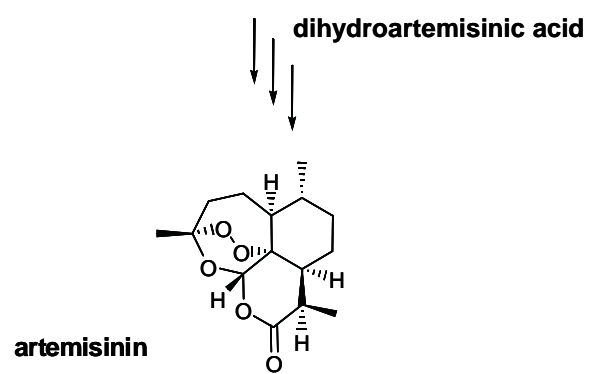

Figure 1. Proposed biosynthetic pathway from farnesyl diphosphate (FDP) to artemisinin. Redrawn from (Bertea et al. 2005) 
expensive multistep process, the plant remains the only commercial source of the drug. However, this compound is present in the leaves and the flowers in only small amounts ranging from 0.01 to $0.8 \%$ of dry weight (Abdin et al. 2003).

Despite the commercial value of artemisinin, until some years ago hardly anything was known about the biosynthesis of artemisinin, particularly about the early enzymatic steps leading to (dihydro)artemisinic acid. Knowledge of the exact biosynthesis of artemisinin should enable us to influence its formation in a direct way, for example by metabolic engineering. Several authors have demonstrated that A. апnиа converts artemisinic (also named arteannuic) acid and dihydroartemisinic (also named dihydroarteannuic) acid to artemisinin (Sangwan et al. 1993a; Wallaart et al. 1999b) (Figure 1). Akhila and coworkers (Akhila et al. 1990) hypothesized a pathway in which the formation from FDP of an unidentified enzyme-bound sesquiterpene-like intermediate represents the first committed step in the biosynthesis of artemisinin.

In addition, many authors have analysed extracts of $A$. annua to search for possible intermediates in the biosynthesis of artemisinin. Artemisinic and dihydroartemisinic acid were reported by many authors, as well as many olefinic mono- and sesquiterpenes and putative intermediates en route from dihydroartemisinic acid to artemisinin (Brown 1994; Jung et al. 1990; Ranasinghe et al. 1993; Wallaart et al. 1999b; Woerdenbag et al. 1993). However, none of the reported olefinic sesquiterpenes seemed to fit in the biosynthetic pathway, nor was a possible intermediate between the sesquiterpene olefin and artemisinic acid ever detected, with the exception of artemisinic alcohol, which was tentatively identified in the roots of A. апnиa (Woerdenbag et al. 1993).

It has been shown by several groups that the cyclization of the ubiquitous precursors geranyl diphosphate, farnesyl diphosphate (FDP) and geranyl geranyl diphosphate to the respective olefinic mono-, sesqui- and diterpene skeletons represents the regulatory step in the biosynthesis of terpenoids (Gershenzon and Croteau 1990; McGarvey and Croteau 1995). The accumulation of artemisinic and dihydroartemisinic acid and the absence of any intermediates en route from FDP to these two compounds support that the first step(s) in the biosynthetic pathway of artemisinin [and again some step(s) from (dihydro)artemisinic acid to artemisinin] are indeed regulatory/rate-limiting. Here we describe the elucidation of the unknown four intermediates and the corresponding five enzymatic steps that constitute the first part of the artemisinin biosynthetic pathway. The implications for strategies to improve artemisinin production are discussed.

\section{ELUCIDATION OF THE BIOSYNTHETIC PATHWAY}

\section{Identification of olefinic sesquiterpenes in A. annua leaves}

Several authors have analysed hydrocarbon sesquiterpenes in A. annua tissues, but a compound that could be the olefinic sesquiterpene intermediate in the biosynthesis of artemisinin was never identified (Ahmad and Misra 1994; Charles et al. 1991; Woerdenbag et al. 1993). In leaf extracts of $A$. annua we detected 14 sesquiterpene 
olefins, of which ten could be identified using GC-MS libraries (Wiley and personal library of W.A. König), retention indices (Adams 1995) and authentic standards (see Figure 2) (Bouwmeester et al. 1999b). Key in being able to demonstrate the

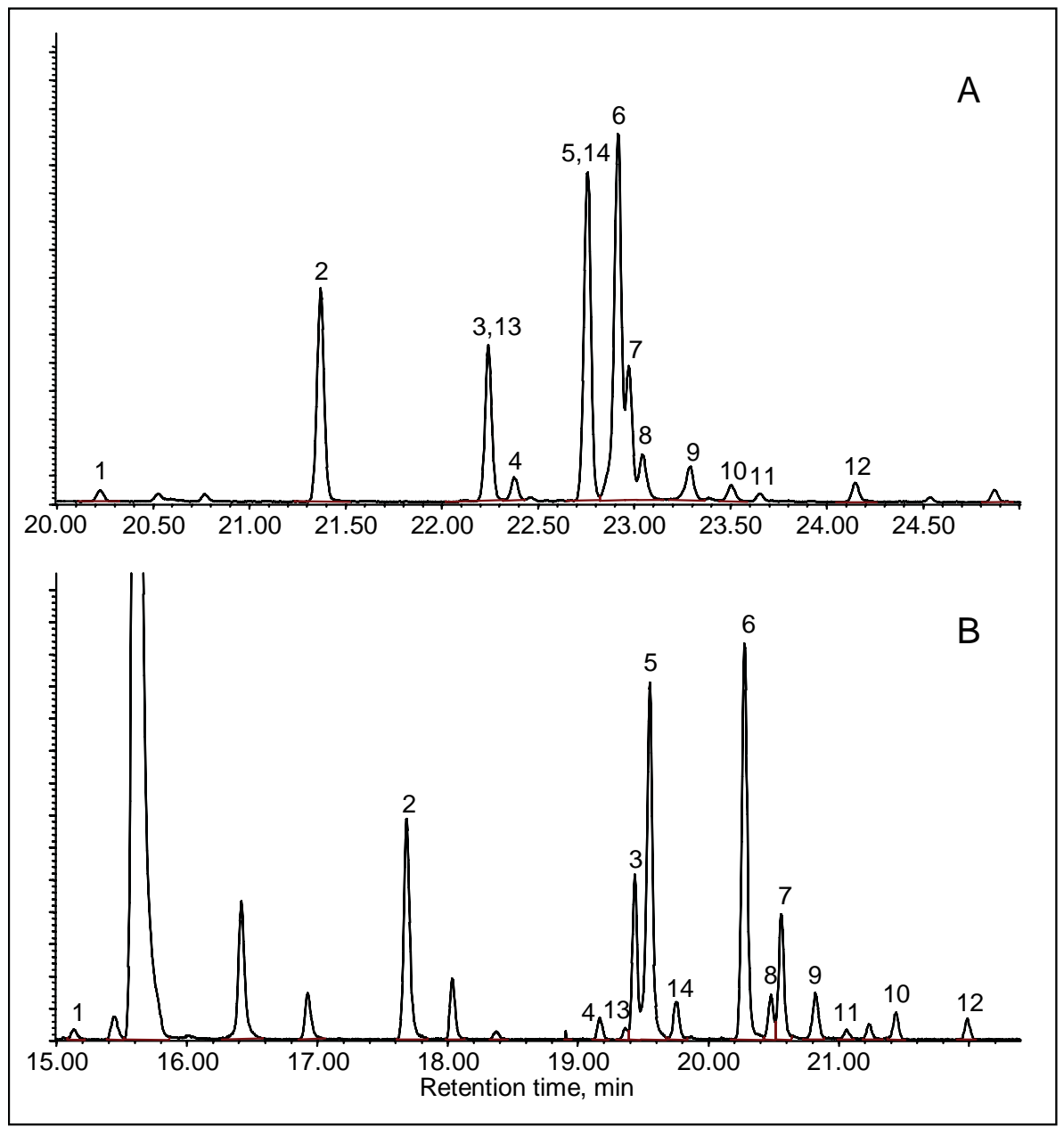

Figure 2. Sesquiterpene olefins in Artemisia annua leaves. Extracts were analysed by GC-MS using a HP 5890 series-II gas chromatograph and HP 5972A Mass Selective Detector equipped with A, an HP-5MS or B, an HP-INNOWax column (both $30 \mathrm{~m}$ x $0.25 \mathrm{~mm}$ i.d., 0.25 $\mu \mathrm{m}$ film thickness). Compounds: 1, $\alpha$-copaene; 2, $\beta$-caryophyllene; 3, trans- $\beta$-farnesene; 4 , muurola-4,11-diene; 5, selina-4,11-diene; 6 , germacrene $D ; 7$, unknown $1 ; 8$, $\beta$-selinene; 9 , bicyclogermacrene; 10, germacrene A; 11, $\gamma$-cadinene; 12, unknown 2; 13, $\alpha$-humulene; 14 , amorpha-4,11-diene. Redrawn from (Bouwmeester et al. 1999b)

presence of compound 14 was that we used two different GC-MS columns. Compound 14 is a minor compound that co-eluted with the major product 5 (selina- 
4,11-diene) on the HP-5MS column but was separated from other sesquiterpenes on the HP-Innowax column. The mass spectrum of compound 14 (see Figure 3) closely resembled the mass spectrum of cadina-4,11-diene (Bohlmann et al. 1984), which

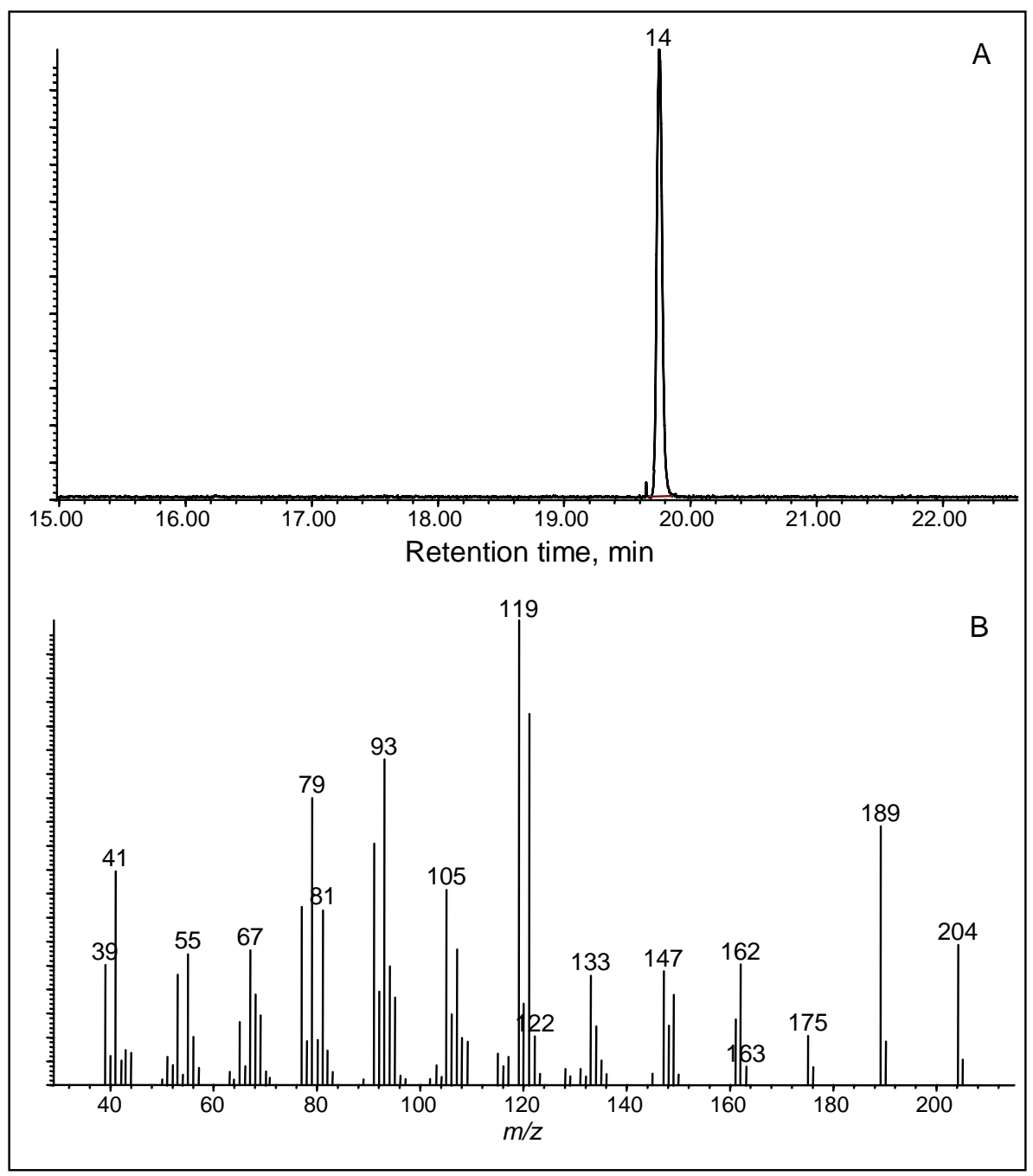

Figure 3. GC-MS analysis of semi-synthetic standard of amorpha-4,11-diene using a HP 5890 series-II gas chromatograph and HP 5972A Mass Selective Detector equipped with an HP-INNOWax column (30 m x 0.25 mm i.d., $0.25 \mu \mathrm{m}$ film thickness). A, chromatogram; B, mass spectrum. From (Bouwmeester et al. 1999b)

was later renamed amorpha-4,11-diene (Connolly and Hill 1991). Considering the structure and configuration, amorpha-4,11-diene is a suitable candidate for the 
olefinic first intermediate in the biosynthesis of artemisinin (Figure 1). The identity of compound 14 was unambiguously proven by semi-synthesis from artemisinic acid. On the HP-INNOwax column compound 14 had the same retention time and mass spectrum as the synthesized product (Figure 3) (Bouwmeester et al. 1999b).

\section{Isolation and characterization of amorpha-4,11-diene synthase}

When a crude enzyme extract of the field-grown A. annua was assayed for sesquiterpene synthase activity with $\left[{ }^{3} \mathrm{H}\right]$-farnesyl diphosphate as a substrate, a range of labelled sesquiterpenes was detected by radio-GC (Bouwmeester et al. 1999b). In order to be able to identify the individual sesquiterpenes (and hence their corresponding synthases) we used partial enzyme purification. Fractional $\left(\mathrm{NH}_{4}\right)_{2} \mathrm{SO}_{4}$ precipitation removed the endogenous sesquiterpenes from the enzyme preparation, and GC-MS analysis of the sesquiterpenes produced by this enzyme preparation upon incubation with FDP showed the presence of the sesquiterpenes $\alpha$-copaene (1), $\beta$-caryophyllene (2), trans- $\beta$-farnesene (3), selina-4,11-diene (5), germacrene D (6), bicyclogermacrene (9), germacrene A (10) and, as one of the major products, amorpha-4,11-diene (14) (Figure 4). Using DEAE anion exchanger batch incubation, $\left(\mathrm{NH}_{4}\right)_{2} \mathrm{SO}_{4}$ precipitation and Mono-Q anion-exchange chromatography (FPLC), amorpha-4,11-diene synthase was further purified. The enzyme preparation thus obtained was used to show that the enzyme had a $\mathrm{K}_{\mathrm{m}}$ of about $0.6 \mu \mathrm{M}$, a molecular mass of $56 \mathrm{kDa}$ and a broad $\mathrm{pH}$ optimum around 6.5-7.0 (Bouwmeester et al. 1999b).

In conclusion, for the first time the presence of amorpha-4,11-diene in A. апnиa was demonstrated. The structure and absolute configuration of this compound make it the likely olefinic sesquiterpene intermediate in the biosynthesis of artemisinin (Figure 1). Moreover, the enzyme activity that catalyses its formation from FDP proved to be the major sesquiterpene synthase activity in A. annua leaf extracts. The fact that amorpha-4,11-diene, in spite of the high in vitro amorpha-4,11-diene synthase activity, can only be detected in minute quantities in the leaf essential oil, strongly suggests that in planta further functionalization of amorpha-4,11-diene occurs.

\section{Biosynthetic pathway beyond amorpha-4,11-diene}

Further modifications of amorpha-4,11-diene to produce the next proven intermediate in artemisinin biosynthesis, artemisinic acid (Sangwan et al. 1993a), can be envisaged to occur analogously to the formation of the diterpenoids kaurenic and abietic acid from kaurene and abietadiene, respectively, and of the sesquiterpenoid germacrene acid from germacrene A (Funk and Croteau 1994; De Kraker et al. 2001; West 1980). The first likely step is the cytochrome-P450catalysed hydroxylation at $\mathrm{C} 12$ of amorpha-4,11-diene yielding artemisinic alcohol. This compound has not been reported in A. annua shoot essential-oil extracts, but was tentatively identified in the roots (Woerdenbag et al. 1993). Subsequently, the alcohol could be oxidized two additional times at $\mathrm{C} 12$ by either cytochrome P450 
enzymes or dehydrogenases, yielding the corresponding acid, artemisinic acid (Funk and Croteau 1994; De Kraker et al. 2001; Sangwan et al. 1993b; West 1980). The C11-C13 double bond of the hence formed artemisinic acid could be reduced

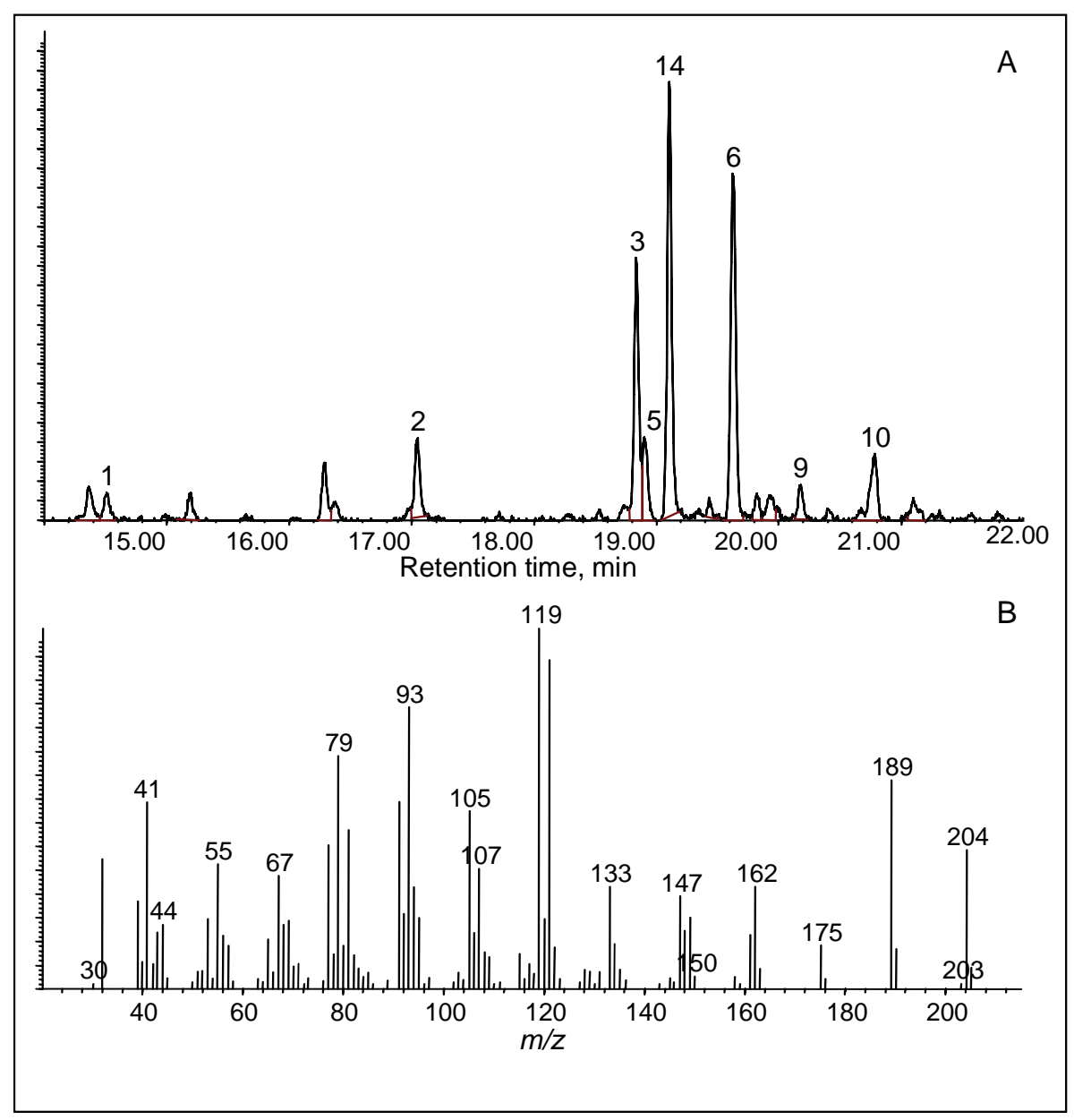

Figure 4. Analysis of the products formed from farnesyl diphosphate by the $\left(\mathrm{NH}_{4}\right)_{2} \mathrm{SO}_{4}$ precipitated enzyme preparation of field-grown Artemisia annua using a HP 5890 series-II gas chromatograph and HP 5972A Mass Selective Detector equipped with an HP-INNOWax column (30 m x $0.25 \mathrm{~mm}$ i.d., $0.25 \mu \mathrm{m}$ film thickness). A, chromatogram of selected ions $\mathrm{m} / \mathrm{z}$ $93+119+161+189+204 ; B$, mass spectrum of compound 14 . Compounds: 1 , $\alpha$-copaene; $2, \beta$ caryophyllene; 3, trans- $\beta$-farnesene; 5 , selina-4,11-diene; 6 , germacrene $D ; 9$, bicyclogermacrene; 10, germacrene A; 14, amorpha-4,11-diene. From (Bouwmeester et al. 1999b)

yielding dihydroartemisinic acid, which was shown by others to be converted non- 
enzymatically to artemisinin (Abdin et al. 2003; Brown and Sy 2004; Wallaart et al. 1999a; Wallaart et al. 1999b). Alternatively, the C11-C13 double-bond reduction may occur in artemisinic alcohol or artemisinic aldehyde, yielding dihydroartemisinic alcohol or dihydroartemisinic aldehyde as intermediates, respectively. To study this unknown part of the pathway, we analysed the presence of putative intermediates and enzymes involved in the conversion of these intermediates in leaves and glandular-trichome extracts of $A$. annua.

Hereto first a number of reference compounds such as artemisinic alcohol, dihydroartemisinic alcohol, artemisinic aldehyde and dihydroartemisinic aldehyde were synthesized - using artemisinic acid and dihydroartemisinic acid as starting materials - or isolated (artemisinic aldehyde). The structures of all isolated or synthesized compounds were confirmed using NMR and MS (Bertea et al. 2005). Subsequently, we looked for these compounds in extracts of $A$. annua leaves as well as in extracts of isolated trichomes. The chromatograms obtained with these two extracts were very similar, indicating that most (if not all) of $A$. annua terpenoids are present in the trichomes (Figure 5). In both cases, artemisinic alcohol, artemisinic aldehyde, artemisinic acid, dihydroartemisinic alcohol, dihydroartemisinic aldehyde, dihydroartemisinic acid and a series of olefinic terpenes were detected (Bertea et al. 2005). Artemisinic acid, dihydroartemisinic acid and the sesquiterpene olefins have been reported before as constituents of A. annua (Bouwmeester et al. 1999a; Wallaart et al. 1999b) but this was the first time artemisinic alcohol, artemisinic aldehyde, dihydroartemisinic alcohol and dihydroartemisinic aldehyde have been identified in A. annua.

In enzyme assays with microsomal pellets of $A$. annua leaf extracts, using amorpha-4,11-diene as a substrate and in the presence of NADPH, we found a small, but consistent amorpha-4,11-diene hydroxylase activity which was absent in the soluble protein fraction $(150,000 \mathrm{~g}$ supernatant), confirming that a cytochrome-P450 enzyme catalyses the formation of artemisinic alcohol from amorpha-4,11-diene. The next putative enzymatic step was assayed by incubating a mixture of artemisinic alcohol and dihydroartemisinic alcohol with the $150,000 \mathrm{~g}$ young-leaf supernatant in the presence of $\mathrm{NAD}^{+} / \mathrm{NADP}^{+}$at $\mathrm{pH}$ 9.0. In the presence of cofactors (Figure 6B), the ratio between artemisinic alcohol and dihydroartemisinic alcohol strongly decreased showing that artemisinic alcohol was converted to artemisinic aldehyde, dihydroartemisinic aldehyde and dihydroartemisinic acid (Bertea et al. 2005). None of these intermediates were formed in the absence of cofactors (Figure 6A). Artemisinic acid was not detected in any of these experiments. To test whether the conversion of dihydroartemisinic aldehyde to dihydroartemisinic acid that was observed in leaf extracts also occurred in trichomes, we incubated the $150,000 \mathrm{~g}$ supernatant of the glandular trichomes with dihydroartemisinic aldehyde in the presence of $\mathrm{NAD}^{+} / \mathrm{NADP}^{+}$. Under these conditions we detected conversion of dihydroartemisinic aldehyde into dihydroartemisinic acid, whereas conversion did not occur in the absence of cofactors.

In order to evaluate all other possible conversions, we also assessed the enzymatic conversion of all putative intermediates in dehydrogenase and reductase assays with and without the appropriate cofactors. Enzymatic oxidation of dihydroartemisinic alcohol to dihydroartemisinic aldehyde and of artemisinic 
aldehyde to artemisinic acid and enzymatic reduction of artemisinic acid to dihydroartemisinic acid did not occur (Bertea et al. 2005). The results of these enzyme assays suggest that the pathway proceeds according to the bold arrows in Figure 1. The chemical reactivity of the intermediates also helps to establish the pathway further. The oxidation of allylic alcohols (like artemisinic alcohol) is much easier than the oxidation of aliphatic alcohols (like dihydroartemisinic alcohol), and
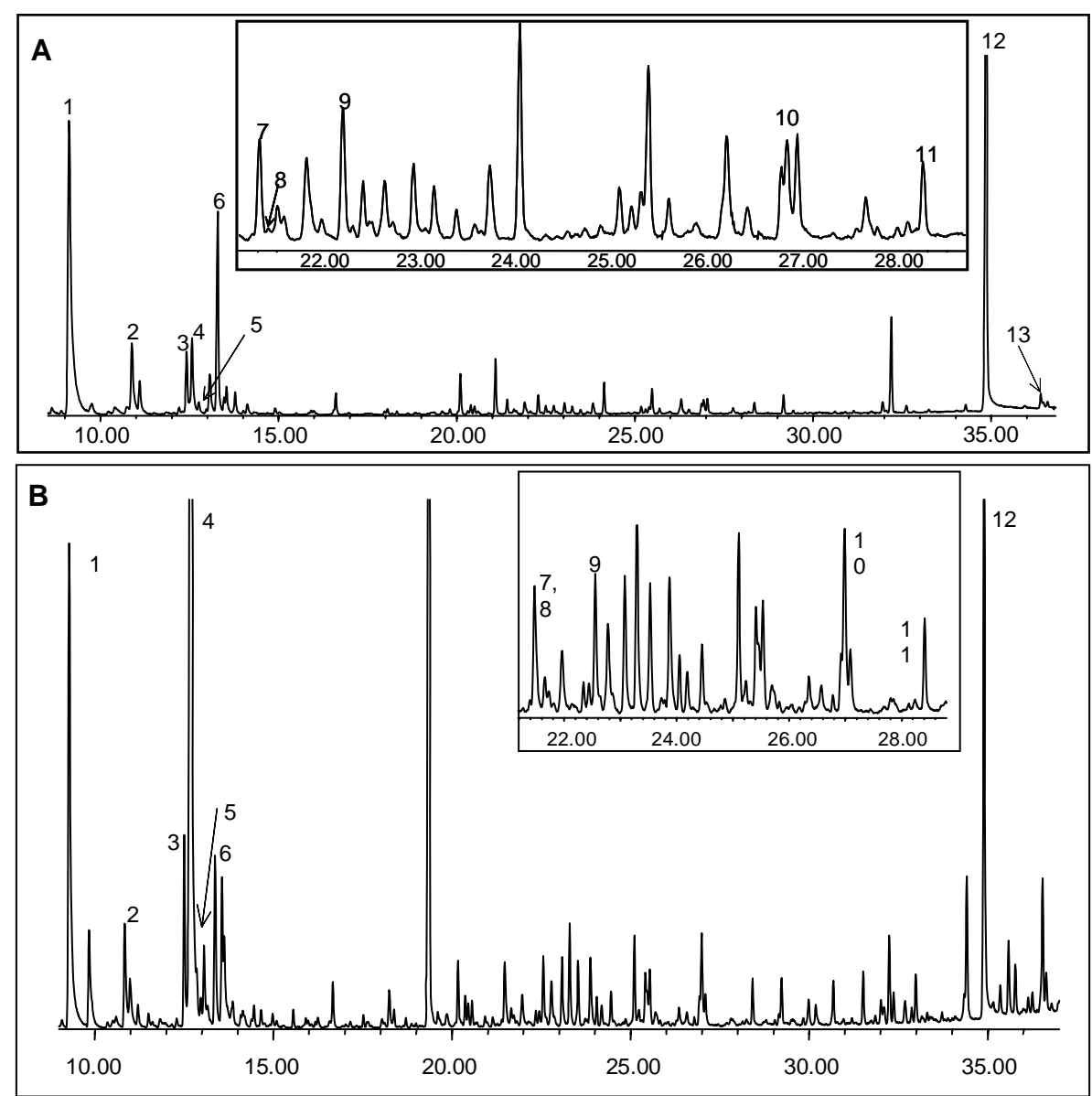

Figure 5. GC-MS analyses (HP-INNOWax column) of essential-oil extracts of greenhousegrown Artemisia annua leaves (A) and glandular trichomes (B). Compounds: 1 = camphor, 2 = germacrene A, 3 = B-farnesene, $4=$ selina-4,11-diene, $5=$ amorpha-4,11-diene, $6=$ germacrene $D, 7$ and 8 = dihydroartemisinic aldehyde (2 diastereomers), 9 = artemisinic aldehyde, $10=$ dihydroartemisinic alcohol, $11=$ artemisinic alcohol, $12=$ dihydroartemisinic acid, 13 = artemisinic acid. From (Bertea et al. 2005)

the reduction of the carbon-carbon double bond is chemically most feasible in an 
$\alpha, \beta$-unsaturated aldehyde (like artemisinic aldehyde) because of Michael-type chemistry (Smith and March 2001). A reduction is also possible in the corresponding $\alpha, \beta$-unsaturated carboxylic acid (like artemisinic acid), but this is not likely to occur in water because the carboxylic acid group is negatively charged at physiological $\mathrm{pH}$, thereby reducing the reactivity of the carbon-carbon double bond towards nucleophiles. Michael type additions are not possible in allylic alcohols, such as artemisinic alcohol, so this compound is the least likely to be reduced.

A

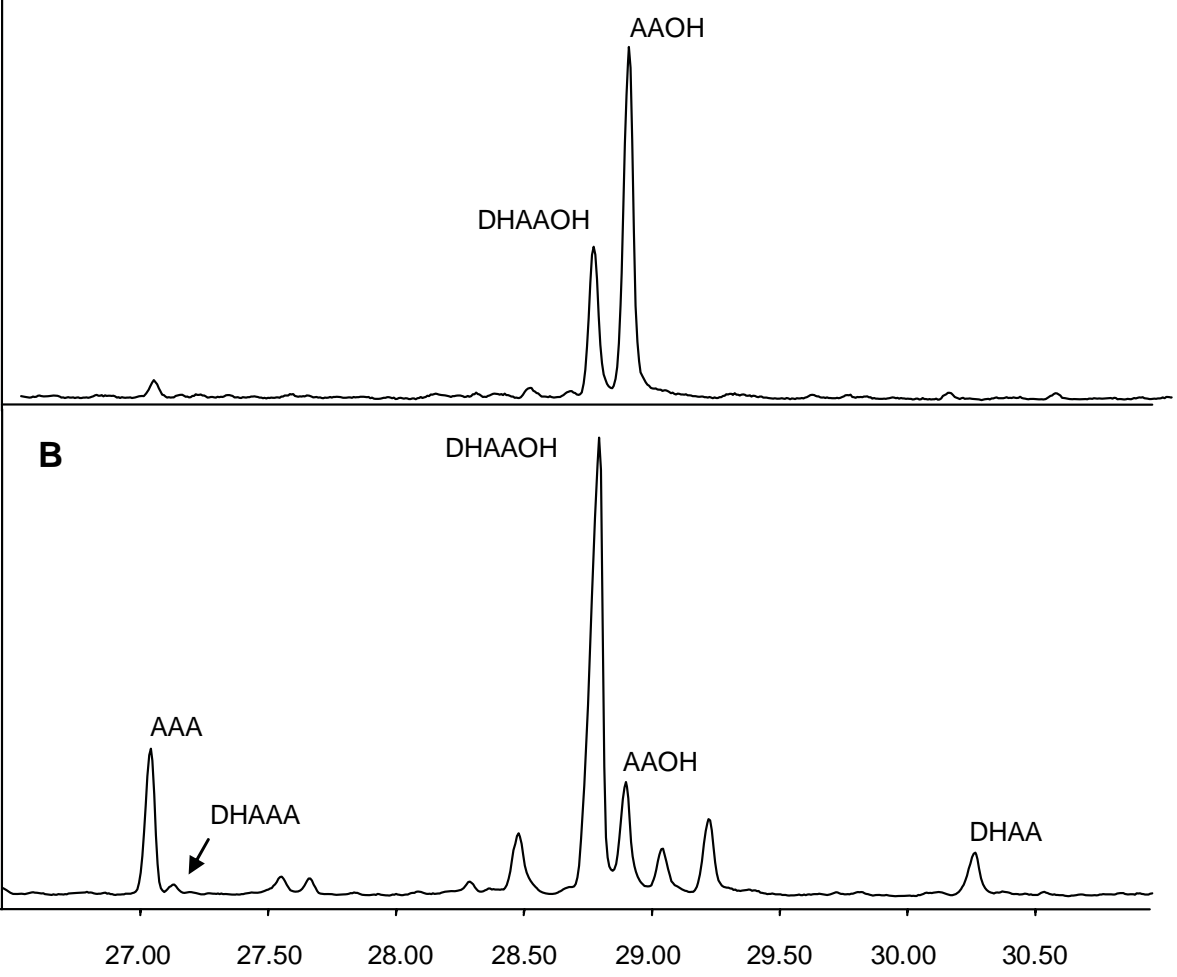

Figure 6. Identification by GC-MS of the products formed in: (A,B) (HP-5MS column) a $150,000 \mathrm{~g}$ supernatant from Artemisia annua leaves incubated with artemisinic alcohol / dihydroartemisinic alcohol at pH 9.0 in the absence of cofactors (A) or in the presence of $N A D^{+}$and $N A D P^{+}(B) . A A O H$ : artemisinic alcohol; $D H A A O H$ : dihydroartemisinic alcohol; AAA: artemisinic aldehyde; DHAAA: dihydroartemisinic aldehyde; DHAA: dihydroartemisinic acid; AA: artemisinic acid. Redrawn from (Bertea et al. 2005)

In conclusion, we identified in A. annua glandular trichome and leaf extracts a number of so far unknown oxidized terpenoids, namely artemisinic alcohol, dihydroartemisinic alcohol, artemisinic aldehyde and dihydroartemisinic aldehyde. 
In a series of enzyme assays we could subsequently demonstrate the enzymatic formation and/or conversion of a number of these putative intermediates: amorpha4,11-diene was oxidized by a putative cytochrome-P450-dependent hydroxylase to artemisinic alcohol (Figure 1, step 2). The next steps in the biosynthetic pathway are the oxidation of artemisinic alcohol at $\mathrm{C} 12$ by a dehydrogenase, yielding artemisinic aldehyde (Figure 1, step 3) and the subsequent reduction of the C11-C13 carboncarbon double bond in artemisinic aldehyde, yielding dihydroartemisinic aldehyde (Figure 1, step 4). Dihydroartemisinic aldehyde is further oxidized to dihydroartemisinic acid in a second dehydrogenase reaction (Figure 1, step 5). Also other authors report that dihydroartemisinic acid is the precursor of artemisinin in $A$. annua (Brown and Sy 2004). These authors also showed that there is no significant desaturation of dihydroartemisinic acid at $\mathrm{C} 11-\mathrm{C} 13$ to yield artemisinic acid, and claim that the reverse reaction, the reduction of artemisinic acid at the $\mathrm{C} 11-\mathrm{C} 13$ position to yield dihydroartemisinic acid, also has never been demonstrated (Brown and Sy 2004). Our biochemical experiments also show that there is no direct enzymatic conversion of artemisinic acid into dihydroartemisinic acid. Hence, we conclude that the route - as shown in bold arrows in Figure 1 - amorpha-4,11-diene $\rightarrow$ artemisinic alcohol $\rightarrow$ artemisinic aldehyde $\rightarrow$ dihydroartemisinic aldehyde $\rightarrow$ dihydroartemisinic acid $\rightarrow$ artemisinin - represents the artemisinin biosynthetic pathway in A. annua.

\section{ISOLATION AND CHARACTERIZATION OF GENES FROM THE BIOSYNTHETIC PATHWAY}

Following the biochemical identification of amorpha-4,11-diene synthase, which catalyses the first dedicated biosynthetic step in artemisinin biosynthesis (Bouwmeester et al. 1999b), the corresponding gene was cloned by several groups almost in parallel (Chang et al. 2000; Mercke et al. 2000; Wallaart et al. 2001) and a patent application was filed (Wallaart and Bouwmeester 1998). Interestingly, the three independently isolated cDNAs are exactly the same suggesting that there is only one copy of this gene present in the plant cell. The heterologous expression of the enzyme allowed to study the mechanism of amorphadiene formation by also studying the formation of side products (Mercke et al. 2000). In this way it was possible to establish that amorpha-4,11-diene synthase first isomerizes farnesyl diphosphate to nerolidyl diphosphate, which is then closed 1,6 to a bisabolyl cation which undergoes 1,10 closure to the final carbon skeleton. Interestingly the $\mathrm{K}_{\mathrm{m}}$ for farnesyl diphosphate as determined with heterologous protein $(0.9 \mu \mathrm{M})$ is virtually the same as the $K_{m}$ reported for the purified protein (Bouwmeester et al. 1999b; Mercke et al. 2000). In the light of a possible use of this gene for alternative production systems for artemisinin, it is interesting to note that Wallaart et al. transformed tobacco with the amorphadiene synthase (Wallaart et al. 2001). This resulted indeed in the production of amorpha-4,11-diene in transgenic plants, but only at trace levels. As we know now, this is not unexpected as since then a number of groups have tried to express sesquiterpene synthases using their natural subcellular targeting, so far without success (Aharoni et al. 2003; Aharoni et al. 
2005). However, recent attempts to boost sesquiterpene production in transgenic plants, e.g. by changing the subcellular targeting to the plastids or even mitochondria, are promising and this problem is expected to be solved in the coming years (Aharoni et al. 2003; Aharoni et al. 2005). A promising step towards fermentative production of artemisinin in micro-organisms is the recent success of Keasling and co-workers, who were able to produce amorpha-4,11-diene in an E. coli strain engineered with the yeast mevalonate pathway and equipped with a synthetic amorpha-4,11-diene synthase cDNA (Martin et al. 2003). However, for heterologous production of artemisinin in plants as well as micro-organisms not only amorpha-4,11-diene synthase is required but also (part of) the other, downstream, steps. Above, we have discussed that we have succeeded in the isolation of glandular trichomes from A. annua and that we detected the presence in these trichomes of all putative pathway intermediates as well as most of the enzymatic activities involved in the artemisinin biosynthetic pathway, including amorpha-4,11diene synthase (Bertea et al. 2005). These results are analogous to results with glandular structures from mint and basil (Gang et al. 2001; Lange et al. 2000), which have also been shown to contain all the enzymatic machinery for essential-oil production.

Based on these preliminary biochemical results, a plasmid cDNA library was made starting from total RNA isolated from glandular trichomes. About 900 of randomly selected clones were partially sequenced and analysed for sequence homologies using the BLASTX algorithm. Fragment assembly identified a total of 459 contigs and the ESTs were then assigned functions based on highest similarity, and categorized into five functional groups (Figure 7). About $47 \%$ of the clones

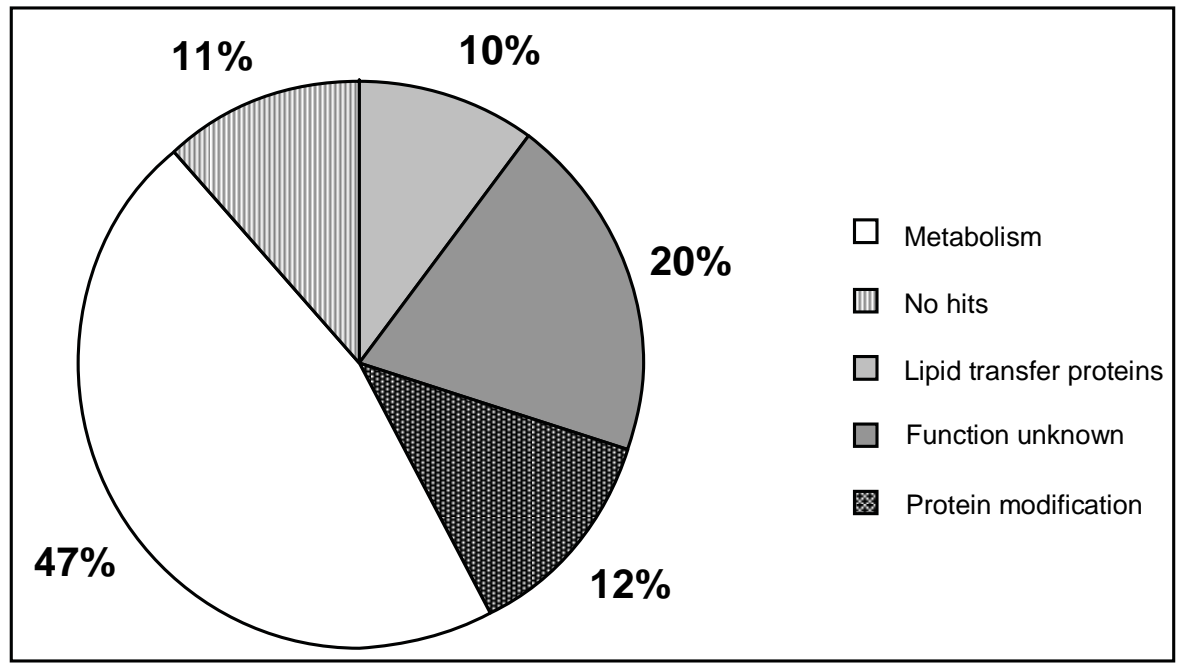

Figure 7. Pie chart showing the representation of 900 ESTs from an Artemisia annua trichome-specific cDNA library over five functional categories. Categories are based on similarity with genes and proteins present in the GenBank 
encoded enzymes involved in primary and secondary metabolism. Up to $6 \%$ of these ESTs encoded enzymes related to isoprenoid biosynthesis (Table 1). Among these clones, amorpha-4,11-diene synthase was found as a singleton, showing that at least the first step in artemisinin biosynthesis is present in the glandular trichomes. Another group of ESTs of high abundance is represented by lipid transfer proteins $(10 \%)$. These proteins are involved in the transport of lipophilic molecules such as essential oils among different cell compartments, and they were found in high abundance also in other glandular trichome cDNA libraries (Gang et al. 2001; Lange et al. 2000).

Table 1. Selection of isoprenoid-biosynthesis-related ESTs and their tentative identification. ESTs are part of a collection of 900 ESTs generated from RNA isolated from glandular trichomes of A. annua. EST identification is based on comparison of the EST sequences obtained with Genbank sequence information. Number of hits indicated how many copies of the particular EST were identified among the 900 that we have sequenced

\begin{tabular}{ll}
\hline EST identification & No. of hits \\
\hline deoxyxylulose 5-phosphate synthase & 3 \\
isopentenyl diphosphate isomerase & 1 \\
geranyl diphosphate synthase (small subunit) & 1 \\
ß-pinene synthase & 3 \\
limonene synthase & 2 \\
linalool synthase & 8 \\
farnesyl diphosphate synthase & 1 \\
germacrene A synthase & 2 \\
amorpha-4,11-diene synthase & 1 \\
other sesquiterpene synthases & 4 \\
\hline
\end{tabular}

\section{CONCLUSIONS}

The above review shows that during the last 6 years an important, missing, part of the pathway by which artemisinin is biosynthesized in the plant $A$. annua has now been elucidated. All the newly identified intermediates, amorpha-4,11-diene, artemisinic alcohol, artemisinic aldehyde and dihydroartemisinic aldehyde, were present in just low concentrations in extracts of leaves as well as trichomes. This is undoubtedly one of the reasons why their discovery has taken such a long time, but also suggests that their formation is one of the rate-limiting steps for the production of artemisinin (another rate-limiting step seems to be the conversion of dihydroartemisinic acid to artemisinin as the former is also often accumulating, see e.g. Figure 5). With the elucidation of the chemistry and biochemistry now the isolation of the corresponding genes has been initiated by a number of groups. With the increasing possibilities of biotechnology, both in plants and in micro-organisms, we expect that new, higher-artemisinin-containing, varieties of $A$. annua as well as, possibly, fermentative production platforms will become available. All this should hopefully make artemisinin available at lower costs to more people in the Third World, so that they who suffer most from malaria can benefit more from this 
valuable and effective drug against this disease.

\section{ACKNOWLEDGEMENTS}

Part of this work was supported by a Marie Curie Individual Fellowship (MCFI2000-52058) to C. M. Bertea.

\section{REFERENCES}

Abdin, M.Z., Israr, M., Rehman, R.U., et al., 2003. Artemisinin, a novel antimalarial drug: biochemical and molecular approaches for enhanced production. Planta Medica, 69 (4), 289-299.

Adams, R.P., 1995. Identification of essential oil components by gas chromatography /mass spectroscopy. Allured Publishing Corporation, Carol Stream.

Aharoni, A., Giri, A.P., Deuerlein, S., et al., 2003. Terpenoid metabolism in wild-type and transgenic Arabidopsis plants. Plant Cell, 15 (12), 2866.

Aharoni, A., Jongsma, M.A. and Bouwmeester, H.J., 2005. Volatile science? Metabolic engineering of terpenoids in plants. Trends in Plant Science, 10 (12), 594-602.

Ahmad, A. and Misra, L.N., 1994. Terpenoids from Artemisia annua and constituents of its essential oil. Phytochemistry, 37 (1), 183-186.

Akhila, A., Rani, K. and Thakur, R.S., 1990. Biosynthesis of artemisinic acid in Artemisia annua. Phytochemistry, 29 (7), 2129-2132.

Bertea, C.M., Freije, J.R., Van der Woude, H., et al., 2005. Identification of intermediates and enzymes involved in the early steps of artemisinin biosynthesis in Artemisia annua. Planta Medica, 71 (1), 40 47.

Bohlmann, F., Gerke, T., Jakupovic, J., et al., 1984. Cadina-4,11-diene from Viguiera oblongifolia. Phytochemistry, 23 (5), 1183-1184.

Bouwmeester, H.J., Konings, M.C.J.M., Gershenzon, J., et al., 1999a. Cytochrome P-450 dependent (+)limonene-6-hydroxylation in fruits of caraway (Carum carvi). Phytochemistry, 50 (2), 243-248.

Bouwmeester, H.J., Wallaart, T.E., Janssen, M.H.A., et al., 1999b. Amorpha-4,11-diene synthase catalyses the first probable step in artemisinin biosynthesis. Phytochemistry, 52 (5), 843-854.

Brown, G.D., 1994. Cadinanes from Artemisia annua that may be intermediates in the biosynthesis of artemisinin. Phytochemistry, 36 (3), 637-641.

Brown, G.D. and Sy, L.-K., 2004. In vivo transformations of dihydroartemisinic acid in Artemisia annua plants. Tetrahedron, 60 (5), 1139-1159.

Butler, D., Maurice, J. and O' Brien, C., 1997. Time to put malaria control on the global agenda. Nature, 386 (6625), 535-540.

Chang, Y.J., Song, S.H., Park, S.H., et al., 2000. Amorpha-4,11-diene synthase of Artemisia annua: cDNA isolation and bacterial expression of a terpene synthase involved in artemisinin biosynthesis. Archives of Biochemistry and Biophysics, 383 (2), 178-184.

Charles, D.J., Cebert, E. and Simon, J.E., 1991. Characterization of the essential oil of Artemisia annua L. Journal of Essential Oil Research, 3, 33-39.

Connolly, J.A. and Hill, R.A., 1991. Dictionary of terpenoids. Vol. 1. Mono- and sesquiterpenoids. Chapman and Hall, London.

De Kraker, J.W., Franssen, M.C.R., Dalm, M.C.F., et al., 2001. Biosynthesis of germacrene A carboxylic acid in chicory roots: demonstration of a cytochrome P450 (+)-germacrene A hydroxylase and $\mathrm{NADP}^{+}$-dependent sesquiterpenoid dehydrogenase(s) involved in sesquiterpene lactone biosynthesis. Plant Physiology, 125 (4), 1930-1940.

Delabays, N., Benakis, A. and Collet, G., 1993. Selection and breeding for high artemisinin (Qinghaosu) yielding strains of Artemisia annua. In: Bernáth, J., Craker, L.E. and Levy, A. eds. WOCMAP I: medicinal and aromatic plants conference. ISHS, Leuven, 203-207. Acta Horticulturae no. 330.

Ferreira, J.F.S., Simon, J.E. and Janick, J., 1995. Developmental studies of Artemisia annua: flowering and artemisinin production under greenhouse and field conditions. Planta Medica, 61 (2), 167-170.

Funk, C. and Croteau, R., 1994. Diterpenoid resin acid biosynthesis in conifers: characterization of two cytochrome P450-dependent monooxygenases and an aldehyde dehydrogenase involved in abietic acid biosynthesis. Archives of Biochemistry and Biophysics, 308 (1), 258-266. 
Gang, D.R., Wang, J., Dudareva, N., et al., 2001. An investigation of the storage and biosynthesis of phenylpropenes in sweet basil. Plant Physiology, 125 (2), 539-555.

Gershenzon, J. and Croteau, R., 1990. Regulation of monoterpene biosynthesis in higher plants. In: Towers, G.H.N. and Stafford, H.A. eds. Biochemistry of the mevalonic acid pathway to terpenoids. Plenum Press, New York, 99-160. Recent Advances in Phytochemistry no. 24.

Gupta, M.M., Jain, D.C., Mathur, A.K., et al., 1996. Isolation of a high artemisinic acid containing plant of Artemisia annua. Planta Medica, 62 (3), 280-281.

Jung, M., El Sohly, H.N. and McChesney, J.D., 1990. Artemisinic acid: a versatile chiral synthon and bioprecursor to natural products. Planta Medica, 56, 624.

Klayman, D.L., 1985. Qinghaosu (artemisin): an antimalarial drug from China. Science, USA, 228 (4703), 1049-1055.

Lange, B.M., Wildung, M.R., Stauber, E.J., et al., 2000. Probing essential oil biosynthesis and secretion by functional evaluation of expressed sequence tags from mint glandular trichomes. Proceedings of the National Academy of Sciences of the United States of America, 97 (6), 2934-2939.

Martin, V.J., Pitera, D.J., Withers, S.T., et al., 2003. Engineering a mevalonate pathway in Escherichia coli for production of terpenoids. Nature Biotechnology, 21 (7), 796-802.

McGarvey, D.J. and Croteau, R., 1995. Terpenoid metabolism. The Plant Cell, 7 (7), 1015-1026.

Mercke, P., Bengtsson, M., Bouwmeester, H.J., et al., 2000. Molecular cloning, expression, and characterization of amorpha-4,11-diene synthase, a key enzyme of artemisinin biosynthesis in Artemisia annua L. Archives of Biochemistry and Biophysics, 381 (2), 173-180.

Ranasinghe, A., Sweatlock, J.D. and Cooks, R.G., 1993. A rapid screening method for artemisinin and its congeners using MS/MS: search for new analogues in Artemisia annua. Journal of Natural Products, 56 (4), 552-563.

Rathore, D., McCutchan, T.F., Sullivan, M., et al., 2005. Antimalarial drugs: current status and new developments. Expert Opinion on Investigational Drugs, 14 (7), 871-883.

Ridley, R.G., 2002. Chemotherapeutic hope on the horizon for Plasmodium vivax malaria? Proceedings of the National Academy of Sciences of the United States of America, 99 (21), 13362-13364.

Rinaldi, A., 2004. Fighting malaria at the crossroads: the tools to battle the disease exist, but the lack of political will in developed nations jeopardizes their success. Embo Reports, 5 (9), 847-851.

Sangwan, R.S., Agarwal, K., Luthra, R., et al., 1993a. Biotransformation of arteannuic acid into arteannuin-B and artemisinin in Artemisia annua. Phytochemistry, 34 (5), 1301-1302.

Sangwan, R.S., Singh-Sanghwan, N. and Luthra, R., 1993b. Metabolism of acyclic monoterpenes: partial purification and properties of geraniol dehydrogenase from lemongrass (Cymbopogon flexuosus Stapf.) leaves. Journal of Plant Physiology, 142 (2), 129-134.

Smith, M.B. and March, J., 2001. March's advanced organic chemistry: reactions, mechanisms and structure. 5th edn. John Wiley, New York.

Van Geldre, E., Vergauwe, A. and Van den Eeckhout, E., 1997. State of the art of the production of the antimalarial compound artemisinin in plants. Plant Molecular Biology, 33 (2), 199-209.

Wallaart, T.E. and Bouwmeester, H.J., DNA encoding amorpha-4,11-diene synthase, (patent).

Wallaart, T.E., Bouwmeester, H.J., Hille, J., et al., 2001. Amorpha-4,11-diene synthase: cloning and functional expression of a key enzyme in the biosynthetic pathway of the novel antimalarial drug artemisinin. Planta, 212 (3), 460-465.

Wallaart, T.E., Pras, N. and Quax, W.J., 1999a. Isolation and identification of dihydroartemisinic acid hydroperoxide from Artemisia annua: a novel biosynthetic precursor of artemisinin. Journal of Natural Products, 62 (8), 1160-1162.

Wallaart, T.E., Van Uden, W., Lubberink, H.G.M., et al., 1999b. Isolation and identification of dihydroartemisinic acid from Artemisia annua and its possible role in the biosynthesis of artemisinin. Journal of Natural Products, 62 (3), 430-433.

West, C.A., 1980. Hydroxylases, monooxygenases and cytochrome P-450. In: Davies, D.D. ed. The biochemistry of plants: a comprehensive treatise. Vol. 2. Metabolism and respiration. Academic Press, New York, 317-364.

Woerdenbag, H.J., Bos, R., Salomons, M.C., et al., 1993. Volatile constituents of Artemisia annua L. (Asteraceae). Flavour and Fragrance Journal, 8 (3), 131-137.

Wright, C.W., 2005. Traditionals antimalarials and the development of novel antimalarial drugs. Journal of Ethnopharmacology, 100 (1/2), 67-71. 